\title{
ESTUDIO DEL NIVEL DE LA MORTALIDAD EN EL PERU SEGUN CAUSAS DE MUERTE, Y LAS PROBABILIDADES FUTURAS DE REDUCCION
}

\author{
Guillermo Vallenas Ochoa
}

\begin{abstract}
RESUMEN.- Se desarrolla un proceso metodológico para analizar los cambios en el nivel de la mortalidad, basados en estimaciones hipotéticas sobre variaciones en la estructura de las causas de muerte, sugiriendo de esta forma, las posibilidades futuras de reducción del nivel de la mortalidad.
\end{abstract}

\section{INTRODUCCION}

En los últimos años el Gobierno viene realizando una serie de acciones orientadas a mejorar las condiciones de vida de la población y disminuir la pobreza que afecta a un gran sector de la misma, con ese propósito se han puesto en marcha Programas Sociales y actividades en Política de Salud en todo el territorio nacional y a no dudar se seguirán realizando acciones de este tipo en el futuro, pues la disminución de la pobreza, el mejoramiento del nivel de salud y por ende la calidad de vida en nuestra población es una tarea permanente y prioritaria.

Es evidente que un aspecto de suma importancia para evaluar las Calidad de Vida de una población es conocer su Estado de Salud, el mismo que tiene incidencia inmediata en la mortalidad, no obstante, se realizan menos esfuerzos para analizar el estado de salud de la población que para la mortalidad, debido posiblemente a la falta de información especializada que permita calcular indicadores sobre la salud; por el contrario, las probabilidades de conocer la mortalidad son mayores, entre otros aspectos porque la muerte es un hecho único, claramente definido que no presenta dudas y por la existencia de estadísticas relativamente confiables, en tanto que los conceptos de salud o enfermedad son a veces imprecisos lo que podría conducir a mediciones bastante vagas, por ello, los estudios sobre la mortalidad general o mortalidad de algunos segmentos de la población como la infantil y la materna, las causas de muerte, etc., constituyen valiosos aportes para determinar el estado de salud de la población y a partir de este conocimiento, formular o rediseñar acciones o políticas de salud cuyo objetivo final sea mejorar las condiciones de vida de la población. 
Por otro lado, el considerar únicamente las mediciones de la mortalidad como indicadores del estado de salud de la población conlleva serios riesgos de desfigurar la realidad, pues en algunos países como el nuestro, donde se registran relativos bajos niveles de mortalidad, los registros de morbilidad muestran inclusive enfermedades re-emergentes, por ello el estudio de las causas de muerte se debe tomar como una parte, no la menos importante, dentro del proceso de transición epidemiológica.

En el país se han elaborado pocos estudios de la mortalidad general basados en las observaciones registradas pese a la existencia de publicaciones presentadas por el MINSA de series anuales de defunciones por edad, sexo y causas de muerte. Por lo general, en los trabajos de proyecciones de población y en otros en los que interviene la mortalidad por grupos de edad y sexo, se acostumbra utilizar Tablas de Mortalidad generadas sobre la base de modelos, las mismas que dan apenas una aproximación de la estructura por edad y sexo de la mortalidad del país.

Es cierto que los registros de defunciones cuya fuente es el Registro Civil están afectados por una omisión cuya magnitud en algunos casos no es conocida, sin embargo, ésta información de la posibilidad de definir estructuras de la mortalidad por edad sexo y principales causas de muerte en nuestro país. Desde que no sólo es importante la estructura sino además el nivel de la mortalidad, se deberá combinar los datos provenientes de censos y/o encuestas con los datos cuya fuente es el registro civil, de forma tal que nos permita construir Tablas de Vida por Edad y Sexo y causas de muerte, las mismas que a no dudar permitirán conocer mejor la situación de la mortalidad del país.

En este estudio se plantea analizar los posibles cambios en el nivel de la mortalidad general del país, basados en estimaciones hipotéticas sobre variaciones en la estructura de las causas de muerte, sugiriendo de esta forma, las posibilidades futuras de reducción del nivel de la mortalidad. La metodología que se utilizará, permite analizar las causas de muerte a través de la técnica de la Tabla de Vida. Según esta técnica se puede ver cómo varía el nivel de la mortalidad según causas de muerte que hipotéticamente se eliminen o disminuyan de acuerdo a metas preestablecidas.

El objetivo pretendido por este tipo de estudios, además de dar a conocer el verdadero nivel y estructura por edad y sexo de la mortalidad del país, sería presentar alternativas de las posibilidades futuras de reducción del nivel de la mortalidad, que sirvan de referencia a los ajustes de metas en los planes y acciones futuras sobre Salud.

No existen en el país trabajos como el presente, en todo caso sería la primera vez que se constituyen Tablas de Mortalidad, al nivel de desagregación propuesto, utilizando los datos sobre causas de muerte del Registro Civil y datos sobre la mortalidad por edad y sexo de la encuesta ENAHO 98-2. 


\section{METODOLOGIA}

Cuando se proponen Programas de Salud Pública dentro de la Política de General de Salud, entre otros aspectos es necesario conocer la repercusión de éstas en el nivel de la mortalidad, una forma analítica de hacerlo es mediante la utilización de técnicas demográficas, consistentes en eliminar teóricamente una, dos o mas causas de muerte, que supuestamente deberían ser controladas por la ejecución de los programas mencionados y estimar el nivel de la mortalidad en estas condiciones. El efecto se mide comparando el nivel inicial de la mortalidad con el nivel resultante en el hipotético caso de eliminación de causas de muerte. Se podría complementar este ejercicio realizando simulaciones sobre cambios futuros de la estructura de las causas de muerte y su impacto en el nivel de la mortalidad, construyendo de esta forma un instrumento técnico de gran valor para tomar decisiones sobre las acciones futuras en el campo de la salud.

El estudio de los cambios del nivel de la mortalidad, basados en el manejo de las causas de muerte, se realiza a través de tres etapas: (a) Estimación de la probabilidad cruda de morir, por una causa especifica, o bien por un grupo de causas, con la presencia de los otros riesgos en la población. (b) Estimación de la probabilidad neta de morir, si se elimina teóricamente una causa específica o un grupo de causas. (c) Medición de los efectos del control de ciertas causas de muerte sobre la esperanza de vida, en el caso hipotético de que se eliminara una causa o un grupo de causas. La Tabla de Mortalidad es el instrumento básico utilizado en este análisis.

Para llevar a cabo este análisis se cuenta con las estadísticas peruanas de defunciones según causas de muerte para los años 1996, 1997 y $1998^{1}$, publicadas por el MINSA, se toman tres años con el objeto de hacer más estable la distribución de las defunciones, es decir, eliminar algunos factores aleatorios que pudieran afectar los registros anuales de defunciones; además, se cuenta con la tabla de mortalidad para el año 1997 elaborada sobre la base de los resultados de la Encuesta Nacional de Hogares del segundo trimestre de 1998 (ENAHO 98-II), cuyo proceso metodológico y análisis han sido publicados por el $\mathrm{INEI}^{2}$, por lo que no se considera en este estudio.

En esta investigación no se da un tratamiento diferencial a la población, según sea menor o mayor de 5 años o de grupo de edades, pese a que se reconoce que las causas de muerte que afectan a la población en los distintos grupos de edades son distintas, debido a la dificultad de contar con información más detallada de la causa de muerte y del tipo de certificación, además este ensayo se considera como una propuesta metodológica para emprender estudios en mayor detalle sobre los efectos de las distintas causas de muerte, que no necesariamente son las planteadas en este trabajo, en grupos de población que muestran grandes diferenciales por edad, sexo y aún por lugar de residencia.

Un aspecto adicional, que tiene relación con la información disponible es que Perú, como otros países en desarrollo, tiene una baja proporción de profesionales en la salud habitante, al margen de la infraestructura sanitaria que también es deficitaria, además, dicha proporción varía notablemente entre una región y otra, aspectos que repercuten notoriamente no solo en la incidencia del tipo de causa de muerte, sino además en la certificación de las causas de muerte.

$1 \mathrm{M}$ inisterio de Salud, Oficina de Estadística e Informática, "Defunciones Registradas Perú : 1996, 1997, 1998", Lima, Perú.

2 Vallenas, Guillermo. "Perú :Mortalidad Infantil, Pobreza y Condiciones de Vida". INEI. Lima - Perú, 1999. 
PERU. TASAS DE RECURSOS HUMANOS DEL SECTOR SALUD-1996

\begin{tabular}{|l|rr|}
\hline \multirow{2}{*}{ DEPARTAMENTO } & \multicolumn{2}{|c|}{ RECURSOS HUMANOS } \\
& (POR 10,000 HABITANTES) \\
& MÉDICOS & ENFERMERAS \\
\hline TOTAL & 10.3 & 6.7 \\
Amazonas & 3.3 & 2.4 \\
Ancash & 6.4 & 3.3 \\
Apurimac & 2.9 & 4.9 \\
Arequipa & 14.5 & 13.8 \\
Ayacacucho & 4.3 & 5.7 \\
Cajamarca & 3.1 & 3.5 \\
Callao & 21.3 & 10.8 \\
Cusco & 5.1 & 6.1 \\
Huancavelica & 2.8 & 2.6 \\
Huanuco & 3.7 & 4.6 \\
Ica & 12.1 & 8.3 \\
Junín & 5.8 & 7.4 \\
La Libertad & 8.3 & 5.6 \\
Lambayeque & 7.8 & 6.3 \\
Lima & 18.9 & 9.1 \\
Loreto & 4.2 & 2.2 \\
Madre de Dios & 9.9 & 9.1 \\
Moquegua & 11.6 & 14.9 \\
Pasco & 5.7 & 8.4 \\
Piura & 6.4 & 3.3 \\
Puno & 3.4 & 4.9 \\
San Martín & 4.3 & 2.5 \\
Tacna & 10.7 & 13.8 \\
Tumbes & 7.0 & 5.7 \\
Ucayali & 4.3 & 4.4 \\
\hline
\end{tabular}

Fuente: Ministerio de Salud

De acuerdo con lo comentado en relación a los recursos humanos e infraestructura sanitaria, hay factores diferenciales propios y externos que se relacionan al conocimiento de la mortalidad por causas, en tal sentido el análisis de la información en el ámbito nacional y por grupos poblacionales y de causas es apenas una especie de aproximación promedio que como tal podría pasar por alto las grandes diferencias internas, en tal sentido, se sugiere la conveniencia de trabajar la incidencia de las causas de muerte en el nivel de la mortalidad, por departamentos o por lo menos por regiones, tomando grupos especiales de población, según la edad y el sexo y por clasificaciones de las causas de muerte que se relacionan con los programas de salud.

\subsection{Probabilidad Cruda de $\operatorname{Morir}\left({ }_{n} Q_{x, \partial}\right)$}

En la probabilidad de morir entre las edades exactas $x$ y $x+n$ por una causa especificada o un grupo de causas, $\partial=\{1,2, \ldots, r\}$ en presencia de los otros riesgos de muerte en la población. 
Una vez corregida la información básica, se procede de la siguiente forma:

Se calcula la distribución porcentual de las muertes, según grandes grupos de causas o causas específicas y edades.

Se aplica la distribución porcentual de las muertes, según edad de la tabla de vida, obteniendo así las defunciones esperadas según causa de muerte o grupos de causas, esto es ${ }_{n} d_{x}={ }_{n} d_{x}^{1}+{ }_{n} d_{x}^{2}+\cdots+{ }_{n} d_{x}^{r}$.

Estas defunciones esperadas se dividen entre el número de sobrevivientes $\left(l_{x}\right)$ a la edad exacta $x$ de la tabla de vida, para obtener la probabilidad cruda de morir $\left({ }_{n} Q_{x, \partial}\right)$ para uno $(r=1)$ o en grupo de causas $(r>1)$, esto es,

$$
\frac{{ }_{n} d_{x}^{\partial}}{l_{x}}={ }_{n} Q_{x, \partial} \quad \partial: \text { Causa o Causas de muerte. }
$$

tal que:

$$
{ }_{n} Q_{x, 1}+{ }_{n} Q_{x, 2}+\cdots+{ }_{n} Q_{x, r}={ }_{n} q_{x}
$$

2.2 Probabilidad Neta de Morir $\left({ }_{n} q_{x, \partial}\right)^{3}$

Es la probabilidad de morir entre las edades exactas $x$ y $x+n$, si se elimina la causa o el grupo de causas.

Una vez obtenida la probabilidad cruda de morir, se obtiene la probabilidad neta de morir a través de:

$$
{ }_{n} q_{x, \partial} \frac{{ }_{n} Q_{x, \partial}}{{ }_{n} q_{n}},
$$

donde:

$$
\begin{aligned}
{ }_{n} P_{x, \partial}=1-{ }_{n} q_{x, \partial}: & \text { Probabilidad neta de sobrevivir entre las edades exactas } x \\
& \mathrm{y} x+n, \text { si se elimina la causa o el grupo de causas. } \\
& : \text { Probabilidad de morir entre las edades exactas } x \mathrm{y} x+n, \\
& \text { y se obtiene de la tabla de vida. } \\
& : \text { Probabilidad de sobrevivir entre las edades exactas } x \mathrm{y} \\
& x+n .
\end{aligned}
$$

${ }^{3}$ Para la deducción de la probabilidad neta de morir véase : Chiang, Chin Long, Introduction to Støchastic Processes in Biostatistics, John Wiley and Sons Inc., Nueva York, 1986. 


\subsection{Supuestos}

Las causas de muerte actúan simultáneamente en cada individuo de la población, existiendo, para cada $\partial$ correspondiente función de intensidad o fuerza de la mortalidad $\mu(t, \partial)$ tal que:

$$
\mu(t, 1)+\cdots \cdots+\mu(t, r)=\mu(t) \text {; siendo } \mu(t) \text { una función del tiempo, " } t \text { ". }
$$

La intensidad del riesgo específico $\mu(t, \partial)$ varía en magnitud absoluta, pero la proporción de esta intensidad respecto al total permanece constante en un intervalo de edad, así :

$$
\frac{\mu_{(t, \partial)}}{\mu_{(t)}}=C_{x, \partial} .
$$

Esta razón es independiente del tiempo, pero es una función del intervalo de edad y de $\partial$.

El supuesto anterior implica lo siguiente:

$$
\frac{\mu_{(t, \partial)}}{\mu_{(t)}}=\frac{{ }_{n} Q_{x, \partial}}{{ }_{n} q_{x}}={ }_{n} q_{x, \partial} .
$$

2.4 Efectos de la Eliminación de las Causas de Muerte en la Esperanza de Vida

Para el cálculo de la esperanza de vida, eliminando una causa específica o un grupo de causas de muerte, es necesario construir una tabla de vida a partir de cada una de las causas de muerte consideradas, o un grupo de causas, partiendo de la probabilidad de sobrevivir calculada anteriormente, y las relaciones siguientes:

\subsection{Funciones de la Tabla de Vida}
i) $l_{x+n}^{\partial}=l_{x} \times{ }_{n} p_{x, \partial}$
ii) $L_{0}^{\partial}=f_{0} \times l_{0}^{\partial}+\left(1-f_{0}\right) \times l_{1}^{\partial}$; donde: $f_{0}=0,23$
iii) ${ }_{4} L_{1}^{\partial}=2 \times\left(l_{1}^{\partial}+l_{5}^{\partial}\right)$
iv) ${ }_{5} L_{x}^{\partial}=2.5 \times\left(l_{x}^{\partial}+l_{x+5}^{\partial}\right)$
vi) $T_{x}^{\partial}=\sum_{x}^{w}{ }_{5} L_{x}^{\partial}$
vii) $e_{x}^{\circ, \partial}=\frac{T_{x}^{\partial}}{l_{x}^{\partial}}$ 


\section{APLICACION DE LA METODOLOGIA}

\subsection{Información Base}

Los cuadros 1, 2 y 3 muestran las defunciones registradas para ambos sexos, por grandes grupos de causas, según edad, en los años 1996, 1997 y 1998. La agrupación se realizó de acuerdo a la clasificación escogida, en la que se destaca la sensibilidad de las causas de muerte a los programas especiales de salud.

En estos cuadros no se realizan correcciones por omisión, dado que la metodología a aplicar no lo requiere, únicamente se realiza la corrección correspondiente al grupo de defunciones cuya edad se desconoce.

\section{Cuadro $\mathrm{N}^{\circ} 1$}

PERU: DEFUNCIONES REGISTRADAS, POR GRUPOS DE CAUSAS, SEGUN EDAD. 1996

\begin{tabular}{|c|c|c|c|c|c|c|}
\hline \multirow{2}{*}{ EDAD } & \multicolumn{7}{|c|}{ DEFUNCIONES POR GRUPOS DE CAUSAS } \\
\cline { 2 - 7 } & TOTAL & GRUPO I & GRUPO II & GRUPO III & GRUPO IV & GRUPO V \\
\hline TOTAL & 92,674 & 23,231 & 14,920 & 13,006 & 9,010 & 32,507 \\
$<01$ & 13,076 & 4,016 & 258 & 33 & 471 & 8,298 \\
$01-04$ & 4,122 & 1,911 & 81 & 111 & 605 & 1,414 \\
$05-09$ & 1,332 & 418 & 64 & 114 & 356 & 380 \\
$10-14$ & 1,092 & 275 & 43 & 124 & 329 & 321 \\
$15-19$ & 1,949 & 461 & 138 & 154 & 667 & 529 \\
$20-24$ & 2,525 & 497 & 182 & 160 & 901 & 785 \\
$25-44$ & 10,824 & 2,121 & 918 & 1,466 & 2,718 & 3,601 \\
$45-49$ & 3,307 & 600 & 433 & 698 & 506 & 1,070 \\
$50-64$ & 13,399 & 2,438 & 2,214 & 3,207 & 1,160 & 4,380 \\
65 y + & 41,048 & 10,494 & 10,289 & 6,939 & 1,297 & 11,729 \\
\hline
\end{tabular}

Fuente: MINSA

\section{Cuadro $\mathrm{N}^{\circ} 2$}

PERU: DEFUNCIONES REGISTRADAS, POR GRUPOS DE CAUSAS, SEGUN EDAD. 1997

\begin{tabular}{|c|c|c|c|c|c|c|}
\hline \multirow{2}{*}{ EDAD } & \multicolumn{7}{|c|}{ DEFUNCIONES POR GRUPOS DE CAUSAS } \\
\cline { 2 - 7 } & TOTAL & GRUPO I & GRUPO II & GRUPO III & GRUPO IV & GRUPO V \\
\hline TOTAL & 89,790 & 22,831 & 14,344 & 13,197 & 9,246 & 30,172 \\
$<01$ & 11,632 & 3,507 & 231 & 17 & 533 & 7,344 \\
$01-04$ & 4,130 & 1,831 & 155 & 96 & 637 & 1,411 \\
$05-09$ & 1,349 & 408 & 44 & 128 & 383 & 386 \\
$10-14$ & 1,084 & 267 & 64 & 124 & 316 & 313 \\
$15-19$ & 1,830 & 390 & 122 & 141 & 709 & 468 \\
$20-24$ & 2,518 & 559 & 174 & 183 & 931 & 671 \\
$25-44$ & 10,627 & 2,141 & 925 & 1,511 & 2,803 & 3,247 \\
$45-49$ & 3,047 & 532 & 358 & 662 & 480 & 1,015 \\
$50-64$ & 12,577 & 2,266 & 2,107 & 3,183 & 1,092 & 3,929 \\
65 y + & 40,996 & 10,930 & 10,164 & 7,152 & 1,362 & 11,388 \\
\hline
\end{tabular}

Fuente: MINSA 
Cuadro $\mathrm{N}^{\circ} 3$

PERU: DEFUNCIONES REGISTRADAS, POR GRUPOS DE CAUSAS, SEGUN EDAD. 1998

\begin{tabular}{|c|c|c|c|c|c|c|}
\hline \multirow{2}{*}{ EDAD } & \multicolumn{7}{|c|}{ DEFUNCIONES POR GRUPOS DE CAUSAS } \\
\cline { 2 - 7 } & TOTAL & GRUPO I & GRUPO II & GRUPO III & GRUPO IV & GRUPO V \\
\hline TOTAL & 91,471 & 25,086 & 15,314 & 13,315 & 9,388 & 28,368 \\
$<01$ & 10,082 & 3,434 & 307 & 18 & 516 & 5,807 \\
$01-04$ & 3,998 & 1,904 & 130 & 95 & 624 & 1,245 \\
$05-09$ & 1,274 & 378 & 74 & 120 & 379 & 323 \\
$10-14$ & 1,052 & 259 & 81 & 121 & 339 & 252 \\
$15-19$ & 1,812 & 393 & 146 & 146 & 693 & 434 \\
$20-24$ & 2,442 & 504 & 169 & 170 & 920 & 679 \\
$25-44$ & 10,411 & 2,136 & 954 & 1,448 & 2,807 & 3,066 \\
$45-49$ & 3,177 & 563 & 375 & 733 & 531 & 975,000 \\
$50-64$ & 12,958 & 2,474 & 2,163 & 3,191 & 1,149 & 3,981 \\
65 y + & 44,265 & 13,041 & 10,915 & 7,273 & 1,430 & 11,606 \\
\hline
\end{tabular}

Fuente: MINSA

En los cuadros procedentes se observa que la estructura anual de las defunciones presenta irregularidades, debido posiblemente, a factores aleatorios típicos en este tipo de información, vinculados a la propia mortalidad y también al registro, centralización de la información y crítica de la certificación de la causa de muerte. Con la finalidad de eliminar estas irregularidades, se procede a formar una estructura promedio para los tres años, la misma que centralizamos en el año 1997, año para el cual se dispone de una Tabla de Vida. El cuadro 4 contiene la información promedio aludida.

\section{Cuadro $\mathrm{N}^{\circ} 4$}

\section{PERU: DISTRIBUCION DE LAS DEFUNCIONES REGISTRADAS, POR GRUPOS} DE CAUSAS, SEGUN EDAD. 1996-98

\begin{tabular}{|c|c|c|c|c|c|c|}
\hline \multirow{2}{*}{ EDAD } & \multicolumn{7}{|c|}{ DEFUNCIONES POR GRUPOS DE CAUSAS } \\
\cline { 2 - 7 } & TOTAL & GRUPO I & GRUPO II & GRUPO III & GRUPO IV & GRUPO V \\
\hline TOTAL & 273,935 & 71,148 & 44,578 & 39,518 & 27,644 & 91,047 \\
$<01$ & 34,790 & 10,957 & 796 & 88 & 1,520 & 21,449 \\
$01-04$ & 12,250 & 5,646 & 366 & 302 & 1,866 & 4,070 \\
$05-09$ & 3,955 & 1,204 & 182 & 362 & 1,118 & 1,089 \\
$10-14$ & 3,228 & 801 & 188 & 369 & 984 & 886 \\
$15-19$ & 5,591 & 1,244 & 406 & 441 & 2,069 & 1,431 \\
$20-24$ & 7,485 & 1,560 & 525 & 513 & 2,752 & 2,135 \\
$25-44$ & 31,862 & 6,398 & 2,797 & 4,425 & 8,328 & 9,914 \\
$45-49$ & 9,531 & 1,695 & 1,166 & 2,093 & 1,517 & 3,060 \\
$50-64$ & 38,934 & 7,178 & 6,484 & 9,581 & 3,401 & 12,290 \\
65 y + & 126,309 & 34,465 & 31,668 & 21,364 & 4,089 & 34,723 \\
\hline
\end{tabular}

Con la finalidad de desdoblar las defunciones de la Tabla de Vida, se calcula la distribución relativa de las defunciones en función de los grandes grupos de causas, tal como se muestran en el cuadro 5 . 


\section{Cuadro $\mathrm{N}^{\circ} 5$ \\ PERU: DISTRIBUCION RELATIVA DE LAS DEFUNCIONES POR GRUPOS DE CAUSAS, SEGUN EDAD. 1996-1998}

\begin{tabular}{|c|c|c|c|c|c|c|}
\hline \multirow{2}{*}{ EDAD } & \multicolumn{7}{|c|}{ DEFUNCIONES POR GRUPOS DE CAUSAS } \\
\cline { 2 - 7 } & TOTAL & GRUPO I & GRUPO II & GRUPO II & GRUPO IV & GRUPO V \\
\hline TOTAL & 100,0 & 26,0 & 16,3 & 14,4 & 10,1 & 33,2 \\
$<01$ & 100,0 & 31,5 & 2,3 & 0,2 & 4,4 & 61,6 \\
$01-04$ & 100,0 & 46,1 & 3,0 & 2,5 & 15,2 & 33,2 \\
$05-09$ & 100,0 & 30,4 & 4,6 & 9,2 & 28,3 & 27,5 \\
$10-14$ & 100,0 & 24,8 & 5,8 & 11,4 & 30,5 & 27,5 \\
$15-19$ & 100,0 & 22,3 & 7,3 & 7,9 & 37,0 & 25,5 \\
$20-24$ & 100,0 & 20,8 & 7,0 & 6,9 & 36,8 & 28,5 \\
$25-44$ & 100,0 & 20,1 & 8,8 & 13,9 & 26,1 & 31,1 \\
$45-49$ & 100,0 & 17,8 & 12,2 & 22,0 & 15,9 & 32,1 \\
$50-64$ & 100,0 & 18,4 & 16,7 & 24,6 & 8,7 & 31,6 \\
65 y + & 100,0 & 27,3 & 25,1 & 16,9 & 3,2 & 27,5 \\
\hline
\end{tabular}

Como se aprecia en el cuadro, el predominio de ciertas causas es diferencial según la edad, así se tiene que, en los menores de 10 años predominan las enfermedades infecciosas y parasitarias como causas de muerte; en el grupo de adultos jóvenes (20 a 44 años) las causas de mayor importancia son las relacionadas con la violencia y accidentes. Finalmente en los adultos mayores son de mayor importancia las muertes relacionadas al cáncer, a enfermedades del corazón y las bronco pulmonares.

Como se mencionó, en este primer trabajo, no se enfoca el carácter diferencial por edad de las causas de muerte, debido a falta de información más desagregada, en tal sentido, las enfermedades que se controlen con la finalidad de ver su efecto en el nivel de la mortalidad, afectarán a todas las edades, aunque en proporciones diferentes según el caso.

\section{Cuadro $\mathrm{N}^{\circ} 6$}

PERU: DISTRIBUCION DE LAS DEFUNCIONES POR GRUPOS DE CAUSAS, SEGUN EDAD. 1996-98

\begin{tabular}{|c|c|c|c|c|c|c|c|c|c|c|c|c|c|}
\hline \multirow{2}{*}{ EDAD } & TOTAL & \multicolumn{3}{|c|}{ GRUPO I } & \multicolumn{3}{|c|}{ GRUPO II } & \multicolumn{3}{|c|}{ GRUPO III } & GRUPO IV & GRUPO V \\
\cline { 3 - 10 } & & TOTAL & A,B,G y H & RESTO & TOTAL & A & RESTO & TOTAL & A y D & RESTO & & \\
\hline TOTAL & 273,935 & 71,148 & 16,460 & 54,688 & 44,578 & 4,262 & 40,316 & 39,518 & 3,419 & 36,099 & 27,844 & 91,047 \\
$<01$ & 34,790 & 10,957 & 2,327 & 8,630 & 796 & 0 & 796 & 68 & 5 & 63 & 1,520 & 21,449 \\
$01-04$ & 12,250 & 5,646 & 2,242 & 3,404 & 366 & 0 & 366 & 302 & 7 & 295 & 1,866 & 4,070 \\
$05-09$ & 3,955 & 1,204 & 357 & 847 & 182 & 0 & 182 & 362 & 21 & 341 & 1,118 & 1,089 \\
$10-14$ & 3,228 & 801 & 244 & 557 & 188 & 4 & 184 & 369 & 43 & 326 & 984 & 886 \\
$15-19$ & 5,591 & 1,244 & 507 & 737 & 406 & 8 & 398 & 441 & 66 & 375 & 2,069 & 1,431 \\
$20-24$ & 7,485 & 1,560 & 744 & 816 & 525 & 16 & 509 & 513 & 41 & 472 & 2,752 & 2,135 \\
$25-44$ & 31,862 & 6,398 & 2,870 & 3,528 & 2,797 & 152 & 2,645 & 4,425 & 600 & 3,825 & 8,328 & 9,914 \\
$45-49$ & 9,531 & 1,695 & 602 & 1,093 & 1,166 & 105 & 1,061 & 2,093 & 309 & 1,784 & 1,517 & 3,060 \\
$50-64$ & 38,934 & 7,178 & 2,046 & 5,132 & 6,484 & 679 & 5,805 & 9,581 & 996 & 8,585 & 3,401 & 12,290 \\
$65 y+$ & 126,309 & 34,465 & 4,521 & 29,944 & 31,868 & 3,298 & 28,370 & 21,364 & 1,331 & 20,033 & 4,089 & 34,723 \\
\hline
\end{tabular}


Desde que en los grupos de causas utilizados para la clasificación inicial hay algunas causas específicas que son factibles de control por acciones de Política de Salud, y otras no, por ejemplo los accidentes, se muestra en el cuadro 6 una clasificación donde figuran algunas causas de muerte que serán materia de eliminación, para ver los efectos en el nivel de la mortalidad.

Con la finalidad de desagregar el total de defunciones de la Tabla de Vida por grupos o causas específicas, se calcula la distribución relativa de las causas de muerte, la cual se muestra en el cuadro 7.

\section{Cuadro $\mathrm{N}^{\circ} 7$}

PERU: DISTRIBUCION DE LAS DEFUNCIONES REGISTRADAS, POR GRUPOS DE CAUSAS, SEGUN EDAD, PARA AMBOS SEXOS. 1997

\begin{tabular}{|c|c|c|c|c|c|c|c|c|c|c|c|c|c|}
\hline \multirow{2}{*}{ EDAD } & \multirow{2}{*}{ TOTAL } & \multicolumn{3}{|c|}{ GRUPO I } & \multicolumn{3}{c|}{ GRUPO I } & \multicolumn{3}{c|}{ GRUPO III } & \multirow{2}{*}{ GRUPO IV } & GRUPO V \\
\cline { 3 - 10 } & & TOTAL & A,B,G y H & RESTO & TOTAL & A & RESTO & TOTAL & A y D & RESTO & \\
\hline TOTAL & 100,0 & 26,0 & 6,0 & 20,0 & 16,3 & 1,6 & 14,7 & 14,4 & 1,2 & 13,2 & 10,1 & 33,2 \\
$<01$ & 100,0 & 31,5 & 6,7 & 24,8 & 2,3 & 0,0 & 2,3 & 0,2 & 0,0 & 0,2 & 4,4 & 61,6 \\
$01-04$ & 100,0 & 46,1 & 18,3 & 27,8 & 3,0 & 0,0 & 3,0 & 2,5 & 0,1 & 2,4 & 15,2 & 33,2 \\
$05-09$ & 100,0 & 30,4 & 9,0 & 21,4 & 4,6 & 0,0 & 4,6 & 9,2 & 0,5 & 8,6 & 28,3 & 27,5 \\
$10-14$ & 100,0 & 24,8 & 7,6 & 14,3 & 5,8 & 0,1 & 5,7 & 11,4 & 1,3 & 10,1 & 30,5 & 27,5 \\
$15-19$ & 100,0 & 22,3 & 9,1 & 13,2 & 7,3 & 0,1 & 7,1 & 7,9 & 1,2 & 6,7 & 37,0 & 25,5 \\
$20-24$ & 100,0 & 20,8 & 9,9 & 10,9 & 7,0 & 0,2 & 6,8 & 6,9 & 0,5 & 6,3 & 36,8 & 28,5 \\
$25-44$ & 100,0 & 20,1 & 9,0 & 11,1 & 8,8 & 0,5 & 8,3 & 13,9 & 1,9 & 12,0 & 26,1 & 31,1 \\
$45-49$ & 100,0 & 17,8 & 6,3 & 11,5 & 12,2 & 1,1 & 11,1 & 22,0 & 3,2 & 18,7 & 15,9 & 32,1 \\
$50-64$ & 100,0 & 18,4 & 5,3 & 13,2 & 16,7 & 1,7 & 14,9 & 24,6 & 2,6 & 22,1 & 8,7 & 31,6 \\
65 y + & 100,0 & 27,3 & 3,6 & 23,7 & 25,1 & 2,6 & 22,5 & 16,9 & 1,1 & 15,9 & 3,2 & 27,5 \\
\hline
\end{tabular}

\subsection{La Tabla de Vida}

El INEI publicó en octubre de 1999, el estudio «Perú: Mortalidad Infantil, Pobreza y condiciones de Vida» [7], uno de cuyos propósitos fue evaluar la suficiencia de la información recolectada en la Encuesta Nacional de Hogares del segundo trimestre de 1998 (ENAHO 98-II), para fines de medición de la mortalidad infantil y mortalidad general. Como uno de los resultados de esta investigación se construyeron para el año 1997 tablas de mortalidad, por sexo, diferenciadas por estratos de pobreza, basadas exclusivamente en la información recolectada en la encuesta sobre defunciones y la población total, ambas según edad.

Para efectos del presente trabajo, se tomó la Tabla de Mortalidad o de Vida, para ambos sexos, que se muestra a continuación. 
Cuadro $\mathrm{N}^{\circ} 8$

ENAHO 98-2: TABLAS DE MORTALIDAD, PARA AMBOS SEXOS. 1997

\begin{tabular}{|c|c|c|c|c|c|c|c|c|}
\hline Edad & $\mathbf{n}$ & $\mathbf{m}(\mathbf{x}, \mathbf{n})$ & $\mathbf{q}(\mathbf{x}, \mathbf{n})$ & $\mathrm{l}(\mathbf{x})$ & $\mathrm{d}(\mathbf{x}, \mathbf{n})$ & $\mathrm{L}(\mathbf{x}, \mathbf{n})$ & $\mathrm{T}(\mathbf{x})$ & $\mathbf{e}(\mathbf{x})$ \\
\hline 0 & 1 & 0,04339 & 0,04188 & 100,000 & 4,188 & 96,521 & $6,491,724$ & 64,92 \\
1 & 4 & 0,00757 & 0,02973 & 95,812 & 2,848 & 376,101 & $6,395,203$ & 66,75 \\
5 & 5 & 0,00214 & 0,01062 & 92,964 & 987 & 462,349 & $6,019,102$ & 64,75 \\
10 & 5 & 0,00140 & 0,00699 & 91,976 & 643 & 458,274 & $5,556,753$ & 60,42 \\
15 & 5 & 0,00155 & 0,00772 & 91,333 & 705 & 454,904 & $5,098,479$ & 55,82 \\
20 & 5 & 0,00202 & 0,01004 & 90,628 & 910 & 450,866 & $4,643,575$ & 51,24 \\
25 & 5 & 0,00267 & 0,01324 & 89,718 & 1,188 & 445,622 & $4,192,708$ & 46,73 \\
30 & 5 & 0,00347 & 0,01721 & 88,530 & 1,524 & 438,843 & $3,747,086$ & 42,33 \\
35 & 5 & 0,00448 & 0,02216 & 87,007 & 1,928 & 430,214 & $3,308,243$ & 38,02 \\
40 & 5 & 0,00580 & 0,02857 & 85,079 & 2,431 & 419,317 & $2,878,029$ & 33,83 \\
45 & 5 & 0,00759 & 0,03724 & 82,648 & 3,078 & 402,546 & $2,458,712$ & 29,75 \\
50 & 5 & 0,01012 & 0,04935 & 79,570 & 3,927 & 388,034 & $2,053,166$ & 25,8 \\
55 & 5 & 0,01378 & 0,06662 & 75,643 & 5,039 & 365,619 & $1,665,132$ & 22,01 \\
60 & 5 & 0,01916 & 0,09143 & 70,604 & 6,455 & 336,882 & $1,299,513$ & 18,41 \\
65 & 5 & 0,02711 & 0,12695 & 64,149 & 8,144 & 300,385 & 962,631 & 15,01 \\
70 & 5 & 0,03885 & 0,17707 & 56,005 & 9,917 & 255,233 & 662,246 & 11,82 \\
75 & 5 & 0,02310 & 0,24601 & 46,088 & 11,338 & 202,096 & 407,013 & 8,83 \\
80 & $\mathrm{w}$ & 0,16958 & 1,00000 & 34,750 & 34,750 & 204,917 & 204,917 & 5,90 \\
\hline
\end{tabular}

Fuente: Guillermo Vallenas, "Mortalidad Infantil y Niveles de Vida". INEI. Lima Perú, 2000.

Desde que la información sobre defunciones considera grupos de edad especiales, se adapta la información de la ENAHO 98-II para modificar la Tabla de Vida, la misma que se muestra como cuadro 9, la cual se utiliza en el presente estudio.

\section{Cuadro $\mathrm{N}^{\circ} 9$}

ENAHO 98-2: TABLA DE MORTALIDAD, PARA AMBOS SEXOS. 1997

\begin{tabular}{|c|c|c|c|c|c|c|c|}
\hline Edad & Intervalo & \multicolumn{7}{|c|}{ FUNCIONES DE LA TABLA DE VIDA } \\
\cline { 3 - 8 } $\mathbf{x}$ & $\mathbf{n}$ & $\mathbf{m}(\mathbf{x}, \mathbf{n})$ & $\mathrm{l}(\mathbf{x})$ & $\mathrm{d}(\mathbf{x}, \mathbf{n})$ & $\mathrm{L}(\mathbf{x}, \mathbf{n})$ & $\mathrm{T}(\mathbf{x})$ & $\mathbf{e}(\mathbf{x})$ \\
\hline 0 & 1 & 0,04188 & 100,000 & 4,188 & 96,521 & $6,491,724$ & 66,92 \\
1 & 4 & 0,02972 & 95,812 & 2,848 & 376,101 & $6,395,203$ & 66,75 \\
5 & 5 & 0,01062 & 92,964 & 987 & 462,349 & $6,019,102$ & 64,75 \\
10 & 5 & 0,00699 & 91,977 & 643 & 458,274 & $5,556,753$ & 60,41 \\
15 & 5 & 0,00772 & 91,334 & 705 & 454,904 & $5,098,479$ & 55,82 \\
20 & 5 & 0,01004 & 90,629 & 910 & 450,866 & $4,643,575$ & 51,24 \\
25 & 20 & 0,07881 & 89,719 & 7,071 & $1,733,996$ & $4,192,709$ & 46,73 \\
45 & 5 & 0,03724 & 82,648 & 3,078 & 405,546 & $2,458,713$ & 29,75 \\
50 & 15 & 0,19380 & 79,57 & 15,421 & $1,090,535$ & $2,053,167$ & 25,8 \\
65 & $\mathbf{w}$ & 1,00000 & 64,149 & 64,149 & 962,632 & 962,632 & 15,01 \\
\hline
\end{tabular}

\subsection{Distribución de las Defunciones según Grupos de Causas de Muerte}

Conocida la distribución relativa de las defunciones, por grupos de causas de muerte, se procede a calcular las defunciones respectivas de la Tabla de Vida, las mismas que serán utilizadas luego para el cálculo de la probabilidades de morir esepecíficas por grupos de causas de muerte. 
Es claro que, luego de la determinación de la defunciones por causas de muerte en la Tabla de Vida, todo el resto metodológico se realiza con estas defunciones, es decir que se calculan las probabilidades de supervivencia, la población estacionaria, el tiempo vivido y la esperanza de vida.

En el cuadro $N^{0} 10$ se muestran las defunciones de la Tabla de Vida, para ambos sexos, desagregadas por causa de muerte.

\section{Cuadro $\mathrm{N}^{\circ} 10$}

PERU: DISTRIBUCION DE LAS DEFUNCIONES DE LA TABLA DE VIDA, POR GRUPOS DE CAUSAS, SEGUN EDAD, PARA AMBOS SEXOS.1997

\begin{tabular}{|c|c|c|c|c|c|c|c|c|c|c|c|c|c|}
\hline \multirow{3}{*}{$\begin{array}{c}\text { EDAD } \\
\mathbf{x}\end{array}$} & INTERVALO & \multicolumn{12}{|c|}{ DEFUNCIONES DE LA TABLA DE VIDA POR GRUPOS DE CAUSAS } \\
\hline & \multirow[b]{2}{*}{$\mathbf{n}$} & \multirow[t]{2}{*}{ TOTAL } & \multicolumn{3}{|c|}{ GRUPOI } & \multicolumn{3}{|c|}{ GRUPO ॥ } & \multicolumn{3}{|c|}{ GRUPO III } & \multirow[t]{2}{*}{ GRUPO IV } & \multirow[t]{2}{*}{ GRUPOV } \\
\hline & & & TOTAL & $A, B, G$ y $H$ & RESTO & TOTAL & A & RESTO & TOTAL & AyD & RESTO & & \\
\hline 0 & 1 & 4.188 & 1.089 & 251 & 838 & 683 & 67 & 616 & $603^{*}$ & 50 & 553 & 423 & 1,390 \\
\hline 1 & 4 & 2,848 & 897 & 191 & 706 & 66 & 0 & 66 & 6 & 0 & 6 & 125 & 1,754 \\
\hline 5 & 5 & 987 & 455 & 181 & 274 & 30 & 0 & 30 & 25 & 1 & 24 & 150 & 327 \\
\hline 10 & 5 & 643 & 195 & 58 & 137 & 30 & 0 & 30 & 59 & 3 & 56 & 182 & 177 \\
\hline 15 & 5 & 705 & 175 & 54 & 121 & 41 & 1 & 40 & 80 & 9 & 71 & 215 & 194 \\
\hline 20 & 5 & 910 & 203 & 83 & 120 & 66 & 1 & 65 & 72 & 11 & 61 & 337 & 232 \\
\hline 25 & 20 & 7,071 & 1,471 & 700 & 771 & 495 & 14 & 481 & 488 & 35 & 453 & 2,602 & 2,015 \\
\hline 45 & 5 & 3.078 & 619 & 277 & 342 & 271 & 15 & 256 & 428 & 58 & 370 & 803 & 957 \\
\hline 50 & 15 & 15.421 & 2.745 & 972 & 1,773 & 1,881 & 170 & 1,711 & 3,393 & 493 & 2,900 & 2,452 & 4,950 \\
\hline 65 & $w$ & 64.149 & 11,803 & 3,400 & 8,403 & 10,713 & 1,091 & 9,622 & 15.781 & 1,668 & 14.113 & 5,581 & 20,271 \\
\hline
\end{tabular}

\subsection{Probabilidad Cruda de Morir}

Esta probabilidad de morir es un índice de la incidencia de cada una de las causas de muerte consideradas por separado, en el nivel de la mortalidad general de la población. Se calcula dividiendo las defunciones por el número de sobrevivientes es decir:

$$
{ }_{n} Q_{x, \partial} \frac{{ }_{n} d_{x}^{\partial}}{l_{x}}
$$

En el cuadro 11, se tienen evidencias claras de cuál es el comportamiento de cada una de estas causas. Así, se nota claramente la importancia de las enfermedades infecto contagiosas en los menores de cinco años, sobre todo en la mortalidad infantil, casi similar al grupo $\mathrm{V}$ o resto de causas (ver apendice). En la población de 65 y más años la mayor incidencia corresponde a las enfermedades degerativas y las cardiovasculares. 


\section{Cuadro $\mathrm{N}^{\circ} 11$ \\ PERU: PROBABILIDAD DE MORIR, POR GRUPOS DE CAUSAS, SEGUN GRUPOS DE EDADES PARA AMBOS SEXOS. 1997}

\begin{tabular}{|c|c|c|c|c|c|c|c|c|c|c|c|c|c|}
\hline \multirow{3}{*}{$\begin{array}{r}\text { EDAD } \\
\times \\
\end{array}$} & \multirow{3}{*}{$\begin{array}{c}\text { INTERVALO } \\
n \\
\end{array}$} & \multicolumn{12}{|c|}{ DEFUNCIONES DE LA TABLA DE VIDA POR GRUPOS DE CAUSAS } \\
\hline & & \multirow[t]{2}{*}{ TOTAL } & \multicolumn{3}{|c|}{ GRUPO I } & \multicolumn{3}{|c|}{ GRUPO ॥ } & \multicolumn{3}{|c|}{ GRUPO III } & \multirow[t]{2}{*}{ GRUPO IV } & \multirow[t]{2}{*}{ GRUPO V } \\
\hline & & & TOTAL & $A, B, G$ y $H$ & RESTO & TOTAL & A & RESTO & TOTAL & A y D & RESTO & & \\
\hline 0 & 1 & 0,04188 & 0,01089 & 0,00251 & 0,00838 & 0,00683 & 0,00067 & 0,00616 & 0,00603 & 0,00050 & 0,00533 & 0,00423 & 0,01390 \\
\hline 1 & 4 & 0,02972 & 0,00936 & 0,00199 & 0,00737 & 0,00069 & 0,00000 & 0,00069 & 0,00006 & 0,00000 & 0,00006 & 0,00130 & 0,01831 \\
\hline 5 & 5 & 0,00699 & 0,00489 & 0,00195 & 0,00294 & 0,00032 & 0,00000 & 0,00032 & 0,00027 & 0,00001 & 0,00026 & 0,00161 & 0,00352 \\
\hline 10 & 5 & 0,00772 & 0,00212 & 0,00063 & 0,00149 & 0,00033 & $0,00000 \mid$ & 0,00033 & 0,00064 & 0,00003 & 0,00061 & 0,00198 & 0,00192 \\
\hline 15 & 5 & 0,01004 & 0,00192 & 0,00059 & 0,00133 & 0,00045 & 0,00000 & 0,00044 & 0,00088 & 0,00010 & 0,00078 & 0.00235 & 0,00212 \\
\hline 20 & 5 & 0,07881 & 0,00224 & 0.00092 & 0,00132 & 0,00073 & 0,00001 & 0,00072 & 0,00079 & 0,00012 & 0,00067 & 0,00372 & 0,00256 \\
\hline 25 & 20 & 0,03724 & 0,01640 & 0,00780 & 0,00860 & 0,00552 & 0,00016 & 0,00536 & 0,00544 & 0,00039 & 0,00505 & 0,02900 & 0,02246 \\
\hline 45 & 5 & 0,03724 & 0,00749 & 0,00335 & 0,00414 & 0,00328 & 0,00018 & 0.00310 & 0,00518 & 0,00070 & 0,00448 & 0,00972 & 0,01158 \\
\hline 50 & 15 & 0,19380 & 0,03450 & 0,01222 & 0,02228 & 0,02364 & 0,00214 & 0,02150 & 0,04264 & 0,00620 & 0,03644 & 0,03082 & 0,06221 \\
\hline 65 & w & 1,00000 & 0,18399 & 0,05300 & 0,13099 & 0,16700 & 0,01701 & 0,14999 & 0,24601 & 0,02600 & 0,22001 & 0,08700 & 0,31600 \\
\hline
\end{tabular}

Es evidente además, que la alta incidencia*de la mortalidad infantil se debe, en su mayor parte, a las enfermedades de la primera infancia, le siguen en importancia las infecciones respiratorias agudas (IRA) y las enfermedades diarreicas agudas (EDA), las mismas que serán controladas para ver su efecto en el nivel de vida.

\subsection{Probabilidad Neta de Morir}

En el cuadro 12 se muestra el proceso de cálculo de la probabilidad neta de morir, para un caso. Como estos pasos son los mismos para todas las causas y edades, basta con mostrar un sólo caso. Veamos cada uno de los elementos de la Tabla.

(2) Probabilidad de sobrevivir por todas las causas: $p(x, n)$ tomadas de la tabla de vida base.

(3) Cálculo del Logaritmo de probabilidad de sobrevivir: $\log (P(x, n))$.

(4) Probabilidad de morir por causa: $Q(x, n, \ell)$.

(5) Probabilidad neta de morir : que es el, cociente de probabilidad cruda de morir por una causa determinada (en este caso las del grupo I) y la probabilidad de morir por todas las causas $\left(q_{x}\right)$ tomada de la tabla de vida base:

$$
q(x, n, \ell)=\frac{Q(x, n, \ell)}{q(x, n)} .
$$

(6) Probabilidad neta de sobrevivir, $P(x, n, \ell)$ calculada como:

$$
P(x, n, \ell)=q(x, n)-q(x, n, \ell)
$$

En la columna (8) de la Tabla se encuentra la probabilidad neta de sobrevivir una vez eliminada la causa.

Finalmente en la columna (9) se obtiene la probabilidad neta de morir luego de eliminar la causa. 
Para estos cálculos se tomaron en cuenta las siguientes combinaciones de causas:

Causa A, B, G y H del Grupo I

Causa A del Grupo II

Causa A y D del Grupo III

Causa A del Grupo II, A y D del Grupo III

Causas Todas las causas involucradas.

Para la selección de las causas, se tomaron en cuenta, según se dijo anteriormente, la importancia numérica y la resistencia al progreso médico.

\section{Cuadro $\mathrm{N}^{\circ} 12$ \\ PERU, PROBABILIDAD NETA DE MORIR ELIMINANDO LAS CAUSAS A, B, G y H DEL GRUPO I AMBOS SEXOS}

\begin{tabular}{|c|c|c|c|c|c|c|c|c|c|c|}
\hline \multirow{4}{*}{$\begin{array}{r}\text { EDAD } \\
\times\end{array}$} & \multirow{4}{*}{$\begin{array}{c}\text { INTERVALO } \\
n\end{array}$} & \multirow{2}{*}{\multicolumn{2}{|c|}{$\begin{array}{l}\text { PROBABILIDAD DE } \\
\text { LA TABLA DE VIDA }\end{array}$}} & \multirow{3}{*}{$\log [p(x, n)]$} & \multicolumn{2}{|c|}{ PROBALIDAD DE MORIR } & \multirow{3}{*}{$\mid \begin{array}{c}\text { PROB.-VIVIR } \\
\text { NETA } \\
p(x, n, 1)\end{array}$} & \multirow{3}{*}{ PRODUCTO } & \multirow{3}{*}{$\begin{array}{c}\text { ANT. - LOG } \\
\text { PROB.-VIVIR } \\
\text { NETA(d) } \\
\end{array}$} & \multirow{3}{*}{$\begin{array}{c}\text { PRO. MORIR } \\
\text { NETA(a) }\end{array}$} \\
\hline & & & & & \multirow{2}{*}{\begin{tabular}{|l|} 
POR CAUSA \\
$Q(x, n, 1)(b)$ \\
\end{tabular}} & \multirow{2}{*}{$\frac{\text { NETA }}{q(x, n, l)(b)}$} & & & & \\
\hline & & $q(x, n)$ & $p(x, n)$ & & & & & & & \\
\hline & & (1) & (2) & (3) & (4) & $(5)=(4) /(1)$ & $(6)=1-(5)$ & $(7)=(3)^{\bullet}(6)$ & (8) & (9) \\
\hline 0 & 1 & 0,01488 & 0,95812 & $-0,01858$ & 0.00251 & 0,05993 & 0,94007 & $-0,017467$ & 0.96058 & 0,03942 \\
\hline 1 & 4 & 0.02972 & 0,97028 & -0.01310 & 0.00199 & 0.06696 & 0,93304 & $-0,012226$ & 0.97224 & 0.02776 \\
\hline 5 & 5 & 0,01062 & 0,98938 & $-0,00464$ & 0,00195 & 0.18362 & 0.81638 & $-0,003786$ & 0.99132 & 0.00868 \\
\hline 10 & 5 & 0,00699 & 0,99301 & -0.00305 & 0.00063 & 0.09013 & 0.90987 & $-0,002771$ & 0,99364 & 0,00636 \\
\hline 15 & 5 & 0,00772 & 0,99228 & $-0,00337$ & 0.00059 & 0,07642 & 0,92358 & $-0,003109$ & 0,99287 & 0,00713 \\
\hline 20 & 5 & 0.01004 & 0.98996 & $-0,00438$ & 0,00092 & 0,09163 & 0,90837 & $-0,003980$ & 0,99088 & 0,00912 \\
\hline 25 & 20 & 0,07881 & 0,92119 & $-0,03565$ & 0,00780 & 0,09897 & 0,90103 & $-0,032123$ & 0,92870 & 0.07130 \\
\hline 45 & 5 & 0,03724 & 0.96276 & -0.01648 & 0,00335 & 0,08996 & 0,91004 & $-0,014999$ & 0,96605 & 0,03395 \\
\hline 50 & 15 & 0,19380 & 0,80620 & -0.09356 & 0,01222 & 0,06305 & 0,93695 & $-0,087658$ & 0,81723 & 0.18277 \\
\hline 65 & $w$ & 1,00000 & 0,00000 & 0 & 0,05300 & 0,05300 & 0,94700 & 0,000000 & 0,00000 & 1,00000 \\
\hline
\end{tabular}

(a) Probabilidad de morir por las causas A, B, G y H del grupo I

(b) Probabilidad neta de morir por las causas A, B, G y H del grupo I.

(c) Probabilidad neta de vivir si se eliminan las causas A, B, G y H del grupo I.

(d) Probabilidad neta de morir si se eliminan las causas A, B, G y H del grupo I.

Cuadro $\mathrm{N}^{\circ} 13$

PERU: PROBABILIDAD NETA DE MORIR AL ELIMINAR ALGUNA CAUSA DE MUERTE AMBOS SEXOS. 1997

\begin{tabular}{|c|c|c|c|c|c|c|}
\hline \multirow{2}{*}{$\begin{array}{c}\text { EDAD } \\
x\end{array}$} & \multirow{2}{*}{$\begin{array}{c}\text { INTERVALO } \\
\text { N }\end{array}$} & \multicolumn{5}{|c|}{ PROBABILIDAD NETA DE MORIR AL ELIMINAR CAUSAS } \\
\hline & & $\begin{array}{l}\text { GRUPO I } \\
\text { A, B, G, H }\end{array}$ & $\begin{array}{c}\text { GRUPO II } \\
\text { A }\end{array}$ & $\begin{array}{c}\text { GRUPO III } \\
\text { A, D }\end{array}$ & $\begin{array}{l}\text { CAUSAS DE } \\
\text { GRUPO I, II }\end{array}$ & $\begin{array}{c}\text { TODAS LAS } \\
\text { CAUSAS }\end{array}$ \\
\hline 0 & 1 & 0,39420 & 0,04122 & 0,04139 & 0,04073 & 0,03827 \\
\hline 1 & 4 & 0,02776 & 0,02972 & 0,02972 & 0,02972 & 0,02776 \\
\hline 5 & 5 & 0,00868 & 0,01062 & 0,01061 & 0,01061 & 0,00867 \\
\hline 10 & 5 & 0,00636 & 0,00699 & 0,00696 & 0,00696 & 0,00633 \\
\hline 15 & 5 & 0,00713 & 0,00771 & 0,00762 & 0,00761 & 0,00702 \\
\hline 20 & 5 & 0,00912 & 0,01003 & 0,00992 & 0,00991 & 0,00899 \\
\hline 25 & 20 & 0,07130 & 0,07866 & 0,07844 & 0,07828 & 0,07076 \\
\hline 45 & 5 & 0,03395 & 0,03706 & 0,03655 & 0,03638 & 0,03308 \\
\hline 50 & 15 & 0,18277 & 0,19188 & 0,18822 & 0,18629 & 0,17516 \\
\hline 65 & $w$ & 1,00000 & 1,00000 & 1,00000 & 1,00000 & 1,00000 \\
\hline
\end{tabular}


En el cuadro 13, se muestra la probabilidad neta de morir, al eliminar las causas o grupos de causas que se consideran en el estudio.

\subsection{Efecto de la Eliminación de una Causa específica en la Esperanza de Vida}

En el análisis del efecto, de la eliminación de una causa o combinaciones de causas en el nivel de la mortalidad, se tomó en cuenta una medida resumen, como es la esperanza de vida, calculada a partir de la probabilidad neta de sobrevivir calculada anteriormente, para ello, se construyen Tablas de Vida, con las probabilidades de morir una vez eliminadas las causas o grupos de causas.

\section{Cuadro $\mathrm{N}^{\circ} 14$ \\ PERU: TABLA DE VIDA ELIMINANDO LA CAUSA A DEL GRUPO II AMBOS SEXOS. 1997}

\begin{tabular}{|c|c|c|c|c|c|c|c|}
\hline Edad & Intervalo & \multicolumn{7}{|c|}{ FUNCIONES DE LA TABLA DE VIDA } \\
\cline { 3 - 8 } $\mathbf{x}$ & $\mathbf{n}$ & $\mathrm{D}(\mathbf{x}, \mathbf{n})$ & $\mathrm{I}(\mathbf{x})$ & $\mathbf{q}(\mathbf{x}, \mathbf{n})$ & $\mathbf{L}(\mathbf{x}, \mathbf{n})$ & $\mathbf{T}(\mathbf{x})$ & $\mathbf{e}(\mathbf{x})$ \\
\hline 0 & 1 & 4,122 & 100,000 & 0,04122 & 96,826 & $6,495,660$ & 64,96 \\
1 & 4 & 2,849 & 95,878 & 0,02972 & 377,814 & $6,398,834$ & 66,74 \\
5 & 5 & 988 & 93,029 & 0,01062 & 462,675 & $6,021,020$ & 64,72 \\
10 & 5 & 643 & 92,041 & 0,00699 & 458,598 & $5,558,345$ & 60,39 \\
15 & 5 & 705 & 91,398 & 0,00771 & 455,228 & $5,099,747$ & 55,8 \\
20 & 5 & 910 & 90,693 & 0,01003 & 451,190 & $4,644,519$ & 51,21 \\
25 & 20 & 7,062 & 89,783 & 0,07866 & $1,725,040$ & $4,193,329$ & 46,71 \\
45 & 5 & 3,066 & 82,721 & 0,03706 & 405,94 & $2,468,289$ & 29,84 \\
50 & 15 & 15,284 & 79,655 & 0,19188 & $1,080,195$ & $2,062,349$ & 25,89 \\
65 & $w$ & 64,371 & 64,371 & 1,00000 & 982,154 & 982,154 & 15,26 \\
\hline
\end{tabular}

\section{Cuadro $\mathrm{N}^{\circ} 15$}

PERU: TABLA DE VIDA ELIMINANDO LAS CAUSAS DE MUERTE, PARA AMBOS SEXOS. 1997

\begin{tabular}{|c|c|c|c|c|c|c|c|}
\hline EDAD & INTERVALO & \multicolumn{6}{|c|}{ FUNCIONES DE TABLA } \\
\cline { 2 - 7 } $\mathbf{X}$ & $\mathbf{N}$ & ENAHO 98 & GRUPO I & GRUPO II & GRUPO III & GRUPO I, II & TODAS \\
\hline 0 & 1 & 64,92 & 66,10 & 64,96 & 65,07 & 65,18 & 66,45 \\
1 & 4 & 66,75 & 67,81 & 66,74 & 66,87 & 66,94 & 68,09 \\
5 & 5 & 64,75 & 65,69 & 64,72 & 64,86 & 64,93 & 65,98 \\
10 & 5 & 60,41 & 61,24 & 60,39 & 60,53 & 60,60 & 61,53 \\
15 & 5 & 55,82 & 56,62 & 55,80 & 55,94 & 56,01 & 56,91 \\
20 & 5 & 51,24 & 52,01 & 51,21 & 51,35 & 51,42 & 52,29 \\
25 & 20 & 46,73 & 47,46 & 46,71 & 46,83 & 46,91 & 47,74 \\
45 & 5 & 29,75 & 30,34 & 29,84 & 29,97 & 30,04 & 30,62 \\
50 & 15 & 25,80 & 26,32 & 25,89 & 26,01 & 26,08 & 26,58 \\
65 & w & 15,01 & 1552,00 & 15,26 & 15,30 & 15,33 & 15,63 \\
\hline
\end{tabular}

En el cuadro 15 se presentan las ganancias logradas en la esperanza de vida una vez eliminada una causa o combinaciones de causas. 


\section{CONCLUSIONES}

Importancia apreciable de las enfermedades infecciosas y parasitarias, sobre todo en las edades menores de 5 años de la población de ambos sexos. Causa D (gripe y bronquitis) con un 5,29 por ciento.

Dentro del grupo I (infecciosas y parasitarias), en las edades mayores de 5 años de la población tienen predominio dos causas específicas: la tuberculosis y la neumonía.

No obstante los triunfos habidos en la lucha contra las enfermedades del grupo I, todavía es alta la proporción de muertes por estas causas. Puede suponer que esta mortalidad es aún mayor debido a la falta de certificación médica y a los errores en la selección de la causa básica que originan las defunciones. De allí que todavía queda un apreciable margen por reducir, que redundaría en una disminución del nivel de la mortalidad.

En las defunciones de la población de los mayores de 5 años predominan las enfermedades del corazón (grupo III), contribuyẹndo a esta proporción los mayores de 45 años de edad, aproximadamente, sin duda, son los que contribuyen a elevar el nivel de la mortalidad de la población peruana.

Llama la atención el apreciable porcentaje de los accidentes y homicidios (grupo IV), con mayor importancia en las edades de 10 a 45 años aproximadamente. A esto contribuye, en gran medida, el proceso de urbanización que está viviendo el país, lo que aumenta el riesgo de morir por accidentes de trabajo y por accidentes de tránsito.

La eliminación hipotética de una causa o un grupo de causas de muerte contribuyen a elevar el nivel de la mortalidad medido a través de la esperanza de vida. Este mejoramiento es diferencial, según sea el tipo de causa o causas que se elimine, y la edad.

En las edades menores de 5 años, las ganancias que se logran son apreciables al eliminar las causas A, B, G y H. Esto nos indica que todavía hay mucho margen para lograr verdaderas mejoras en el nivel de la mortalidad de la población, dependiendo ellas de los esfuerzos tendientes a mejorar la atención en los servicios descentralizados de salud, dando énfasis en la salud preventiva de enfermedades que provocan la alta frecuencia de muertes en las primeras edades.

Las estimaciones obtenidas nos dan un marco teórico para la evaluación de los programas sanitarios tendientes a combatir las más importantes causas de muerte de la población, en un plazo de 5 ó 10 años.

Si consideramos la población adulta, las cifras nos indican que las mayores ganancias se logran en las enfermedades del corazón (grupo III), siguiéndole en importancia las ocasionadas por accidentes y homicidios (grupo IV) y por cáncer (grupo II). Dentro de estos tres grupos de causas de muerte, las que a corto plazo pueden contribuir al aumento de la esperanza de vida podrían ser las del grupo IV, ya que los accidentes pueden ser atenuados mediante medidas preventivas en los centros de trabajo, y mayor vigilancia en las carreteras, igualmente algunos tipos de cáncer que pueden ser detectados sobre la base de campañas de control. 
Las ganancias hipotéticas que pueden lograrse al eliminar las enfermedades seleccionadas en todos los grupos (menos las del grupo IV) son factibles de control sobre la base de la mejora de la calidad de atención de los servicios públicos de salud, la mayor cobertura de los mismos en el territorio nacional y aceptación de los progresos médicos en este tipo de enfermedades, sobre todo en las degenerativas y las bronco pulmonares.

\section{BIBLIOGRAFIA}

[1] Alvarado, Ricardo. "Análisis de la mortalidad por causas", 1960. CELADE, Santiago de Chile (inédito).

[2] Arrias Adalberto. "Análisis de la Mortalidad por Causas”. CELADE, 1961.

[3] Cerisola, M. J. Elsa, República. "Análisis de la mortalidad por causas", 1960. CELADE, Serie C, No 109, Santiago de Chile, 1968.

[4] Chiang, Chin Long. "Introduction to Stochastic Processes in Biostatistics". John Wiley and Sons, 1968.

[5] Elizaga, Juan Carlos. "Métodos Demográficos para el Estudio de la Mortalidad". CELADE, Serie E, No 4, Santiago de Chile, 1969.

[6] Vallenas, Guillermo. "Construcción de Indicadores Demográficos en base a Censos y En-cuestas”. INEI, Lima-Perú, 2000.

[7] Vallenas, Guillermo. "Mortalidad Infantil, Pobreza y Condiciones de Vida". INEI, Lima-Perú, 1999.

[8] U.S. Departamento of Health Education and Welfare, United States Life Tables by Cau-ses of Death: 1959-61. Washington D.C., 1968. 


\section{APENDICE}

\section{GRUPO I: Enfermedades Infecciones y Parasitarias, Gripe y Neumonías}
A. Enfermedades Infecciosas Intestinales.
B. Tuberculosis.
C. Otras enfermedades Bacterianas.
D. Enfermedades Virales.
E. Otras enfermedades transmitidas por Artrópodos.
F. Enfermedades Venéreas.
G. Otras Infecciosas y Parasitarias.
H. Enfermedades de las Vías Respiratorias.
I . Otras Enfermedades del Aparato Respiratorio.

\section{GRUPO II: Enfermedades cardiovasculares y renales}
A. Enfermedades Hipertensiva.
B. Enfermedad Isquémica del Corazón.
C. Enfermedad Cardiovascular.
D. Otras Enfermedades del Aparato Circulatorio.

\section{GRUPO III: Neoplasma Malignos}
A. Tumor Maligno del Labio de la Cavidad Bucal y de la Faringe.
B. Tumor Maligno de Otros Organos Digestivos y del Peritoneo.
C. Tumor Maligno de los Organos Respiratorios e Intra toráxicos.
D. Tumor Maligno de los Huesos, del Tejido Conjuntivo, de la Piel y de la Mama.
E. Tumor Maligno de los Organos Genitourinarios.
F. Tumor Maligno de otros sitios y los no especificados.
G. Tumor Maligno del Labio de la Cavidad Bucal y de la Faringe.
H. Enfermedades del Tejido Linfático y de los Organos Hematopoyéticos.
I . Carcinoma In Situ.

\section{GRUPO IV: Accidentes y Violencia}
A. Fractura.
B. Luxaciones, Esguinces y desgarros.
C. Traumatismos Internos e Intracraneales, incluso los de los nervios.
D. Heridas y Traumatismos de los Vasos Sanguíneos.
E. Efectos de Cuerpo Extraño que penetra por un Orificio Natural.
F. Quemaduras.
G. Complicaciones de la atención Médica y Quirúrgica.
H. Otras lesiones y complicaciones precoces de los Traumatismos.
I . Efectos tardíos de Traumatismos, Envenenamiento de efectos Tóxicos y otras causas externas.

\section{GRUPO V: Resto de causas}

Aun cuando es esta agrupación se pierde la especifidad, en cierta forma, está acorde con las principales causas de muerte de la población. Es indudable que se debería especificar mucho más las causas, sobre todo según grupos 
de edades, desde que la incidencia de ciertas enfermedades que conducen a la muerte, es diferencial según la edad, por ejemplo en niños menores de 5 años las enfermedades diarreicas agudas y las infecciones respiratorias agudas son las principales causas de muerte, en la medida que desciende el nivel de la mortalidad infantil, las causas endógenas en los menores de un año tomarán mayor importancia. Del mismo modo en la población adulta, joven, los accidentes o muerte violenta son las de mayor incidencia, finalmente en los adultos viejos, no cabe duda que las enfermedades degenerativas y las bronco pulmunares tienen mucha importancia.

La profundización de este estudio debería tomar en cuenta causas específicas de una clasificación mayor, donde claramente se discrimine los grupos de edad y el sexo de la población, así como el lugar de residencia. 